\title{
A Turbo Iteration Algorithm In 16QAM Hierarchical Modulation
}

\author{
$\mathrm{Xu}$ Zhe \\ Electric and Information College \\ North-Western Poly-Technology University \\ Xi' an China \\ xuzhe0330@163.com \\ Wang YongSheng \\ Electric and Information College \\ North-Western Poly-Technology University \\ Xi' an China
}

\author{
Florence Alberge \\ Division signaux \\ Supelec ecole \\ Paris orsay France \\ Pierre DUHAMEL \\ Division signaux \\ Supelec ecole \\ Paris orsay France
}

\begin{abstract}
In the next generation communication protocol "DVBSH", it offers a new transmitting scheme based on the turbo code, OFDM modulation and 16QAM hierarchical modulation to increase the flexibility in the information delivery. This scheme is using Hierarchical modulation to enhance the UE (user equipment) performance which is in the center of a sector and also ensure the basic service when $U E$ move to the region of a sector. Hierarchical modulation uses two different bit streams on to different constellation of the constellation map. This scheme ensures two different bit streams have corresponding BER performance and then bring on the flexibility to the transmitting service. The simulation results indicates that in the Rayleigh and Gaussian channel, this scheme can gain a very high BER performance of high priority bit stream, and in the high SNR, it can also gain a high BER performance of low priority bit stream.
\end{abstract}

Keywords- turbo iteration; soft-input soft-output; hierarchical modulation; OFDM

\section{INTRODUCTION}

At $14^{\text {th }}$ February 2007, the DVB Steering Board has approved the DVB-SH(satellite services to handhelds) specification in Geneva at the $53^{\text {rd }}$ meeting of the DVB Steering Board[1]. DVB-SH includes features such as turbo coding, OFDM modulation and hierarchical modulation to ensure transmission of high quality video information, at the same time it can also take account of the low quality information when the SNR condition is good enough. Since the handhelds terminals are always in the moving conditions, so the hierarchical modulation can satisfied this users who are in the center of one sector to receive high quality signals(high priority bits\& low priority bits), when the user is moving to the region of one sector, it can ensure receiving the basic signals(high priority bits).

The model discussed in this paper is a turbo code with 16QAM-OFDM system which based on the DVB-SH scheme. The turbo code has excellent performance by using the turbo iteration. But in the traditional schemes with Gray constellation mapping, the total performance of iteration is not used up. If designed properly, we can gain better performance. So not only the DVB project but also LTE project are interested in this technology now[2-4].

This paper discusses a new iteration scheme which mixed with the hierarchical modulation. It can achieve an additional gain compare to the traditional schemes. With the different modulation factor $(\alpha=1,2,3)$ and different modulation mapping, the BER performance are different and the BER performance of the novel modulation ideal is better than which of the traditional mapping.

\section{SCHEME DESCRIPTION}

\section{A. modulation scheme}

Figure 1 shows the system simulation scheme. The feature blocks include turbo encoder, hierarchical modulation and OFDM modulation. At the turbo encoder port, the HP (high priority bit stream) bits and the LP (low priority bit stream) bits are separated and inputted into two turbo encoders, then are interleaved. After that, the two bit streams are fixed together and sent to the modulator, in the modulator, they are mapped onto the respective constellation location. The OFDM modulation block consists of S/P block, IFFT block and CP block. OFDM demodulation block consists of $\mathrm{CP}$ deleting block, P/S block, and FFT block. The simulation processing is supposed to be synchronized.

\section{B. Algorithm Descriptions}

\section{1) Turbo decoding algorithm}

The turbo code principle is provided by C.Berrou at ICC'93 meeting firstly, after a fast developing, now there are several decoding algorithm like: MAP algorithm, soft-Viterbi algorithm and so on[5]. In [5-8], it provides the design principles of turbo code and the iteration decoding method. In the MAP decoder, it decides the log a posterior probability

(LAPP) ratio defined as ${ }^{[9]}$ : 


$$
L\left(u_{t}\right)=\log \left(\frac{p\left(u_{t}=1 \mid y\right)}{p\left(u_{t}=0 \mid y\right)}\right)
$$

Incorporating the code's trellis, this may be written as:

$$
L\left(u_{t}\right)=\log \left(\frac{\sum_{S \in 1} p\left(s_{k-1}=s^{\prime}, s_{k}=s, y\right) / p(y)}{\sum_{S \in 0} p\left(s_{k-1}=s^{\prime}, s_{k}=s, y\right) / p(y)}\right)
$$

Consider (2), if we want to calculate the value of $L\left(u_{t}\right)$, first we need to compute $p\left(s^{\prime}, s, y\right)=p\left(s_{k-1}=s^{\prime}, s_{k}=s, y\right)$, in BCJR algorithm [7] for doing this is:

$$
p\left(s^{\prime}, s, y\right)=\alpha_{t-1}\left(s^{\prime}\right) \cdot \gamma_{t}\left(s^{\prime}, s\right) \beta_{t}(s)
$$

we define the modified probabilities:

$$
\begin{aligned}
\tilde{\alpha}_{t}(s) & =\alpha_{t}(s) / p\left(y_{1}^{t}\right) \\
\text { and } \quad \tilde{\beta}_{t}(s) & =\beta_{t}(s) / p\left(y_{t+1}^{\tau} \mid y_{1}^{t}\right)
\end{aligned}
$$

In summary, we modify BCJR-MAP algorithm by using (2) and (3) to obtain:

$$
L\left(u_{t}\right)=\log \left(\frac{\sum_{S \in 1} \tilde{\alpha}_{t-1}\left(s^{\prime}\right) \cdot \gamma_{t}\left(s^{\prime}, s\right) \cdot \widetilde{\beta}_{t}(s)}{\sum_{s \in 0} \tilde{\alpha}_{t-1}\left(s^{\prime}\right) \cdot \gamma_{t}\left(s^{\prime}, s\right) \cdot \tilde{\beta}_{t}(s)}\right)
$$

we can deduct that $\gamma_{t}\left(s^{\prime}, s\right)$ has the direct ratio to[10]:

$$
\gamma_{t}\left(s^{\prime}, s\right) \propto e^{-\frac{1}{2 \sigma^{2}}|y-h|^{2}} P\left(s \mid s^{\prime}\right) \gamma_{d e c}(u \mid y)
$$

where $e^{-\frac{1}{2 \sigma^{2}}|y-h s|^{2}}$ is the information from the channel, and $P\left(s \mid s^{\prime}\right)$ is the probability from state $s$ transform to $s^{\prime}$, and $\gamma_{\text {dec }}(u \mid y)$ is the extrinsic probability for receive data $u$ from the symbol $y$.

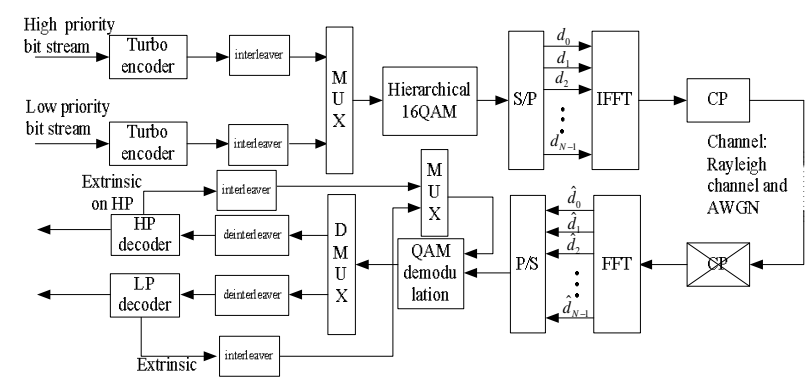

Figure 1 hierarchical modulation simulation scheme

2) Modulation Mapping

The modulation mapping scheme is depicted in reference [1]. When the 16QAM modulation is used in the modulation block, and when the modulation factor $\alpha=1,2,4$, the modulation mapping is shown in figure 2. the normalize factor of the three mapping is $\sqrt{10}, \sqrt{20}, \sqrt{52}$.

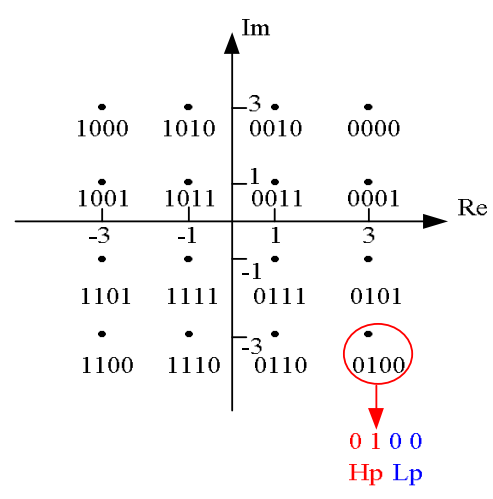

(a) $\alpha$ equal to 1

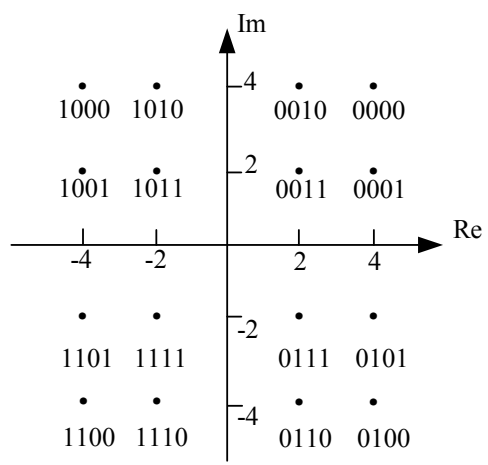

(b) $\alpha$ equal to 2

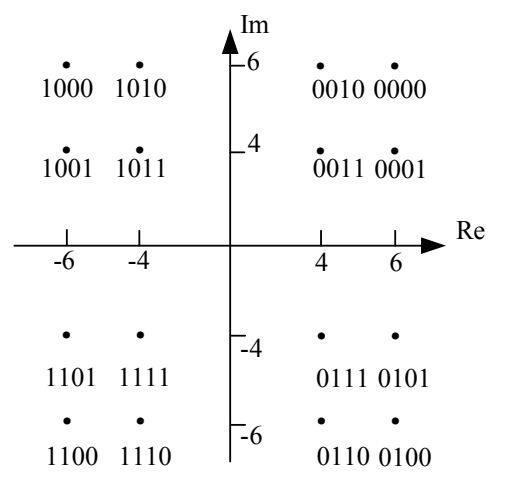

(b) $\alpha$ equal to 4

Figure 2 16QAM hierarchical modulation

The modulation location of Hp bits and Lp bits are depicted in figure 2 (a). This mapping scheme is different to the traditional gray mapping, we can see that the four symbols in every quadrant have the same Hp bits but different Lp bits, this is also called constellation overlapping in reference [2] which ensure the $\mathrm{Hp}$ bits to be transmitted correctly.

\section{3) De-modulator to Decoder Iteration Algorithm}

Considering on the mapping constellation given at section 2.2.2, there are four bits in each mapping symbol. So the APP of one bits in a symbol can be depicted as ${ }^{[10]}$ :

$$
\begin{aligned}
P\left(d_{k}^{1} \mid y_{k}\right) & =\sum_{d_{k}^{2}, d_{k}^{3}, d_{k}^{4} \in\{0,1\}} P\left(d_{k}^{1} \ldots d_{k}^{4} \mid y_{k}\right) \\
& =\sum_{d_{k}^{2}, d_{k}^{3}, d_{k}^{4} \in\{0,1\}} \frac{P\left(y_{k} \mid d_{k}^{l} . . . d_{k}^{4}\right) P\left(d_{k}^{1} \ldots d_{k}^{4}\right)}{P\left(y_{k}\right)}
\end{aligned}
$$


Where $d_{k}^{1}, d_{k}^{2}, d_{k}^{3}, d_{k}^{4}$ is the four bits in symbol $y_{k}$. Since we consider the interleaver to be ideal, $d_{k}^{1}, d_{k}^{2}, d_{k}^{3}, d_{k}^{4}$ is independent. So (6) can be reformulated as:

$$
P\left(d_{k}^{1} \mid y_{k}\right) \approx \sum_{d_{k}^{2}, d_{k}^{3}, d_{k}^{4} \in\{0,1\}} \frac{P\left(y_{k} \mid d_{k}^{l} . \quad . d_{k}^{4}\right) P\left(d_{k}^{1}\right) \ldots P\left(d_{k}^{4}\right)}{P\left(y_{k}\right)}
$$

Which can be expressed in the case of a white Gaussian noise $n_{k}$ with zero mean and variance $\sigma^{2}$ and under the Rayleigh fading process of $h_{k}$ :

$$
P\left(d_{k}^{1} \mid y_{k}\right) \propto \sum_{d_{k}^{2}, d_{k}^{3}, d_{k}^{4} \in\{0,1\}} e^{-\frac{1}{2 \sigma^{2}}\left|y_{k}-h_{k} s\left(d_{k}^{1}, \ldots, d_{k}^{4}\right)\right|^{2}}
$$

Now we have known that Formula (5) has given a way to get the MAP decoding results. Re-formula (5) in the new condition, we can get:

$$
\gamma_{t}\left(m^{\prime}, m\right)=\frac{P\left\{c_{t}^{\prime}\left(m^{\prime}, m\right) ; c_{t}^{2}\left(m^{\prime}, m\right)\right\} P\left(y_{k}||_{\left.\pi_{t}^{\prime}\right)} P\left(y_{k}||_{\left.\pi_{t}^{2}\right)}\right)\right.}{P\left\{c_{t}^{(}\left(m^{\prime}, m\right) ; c_{t}^{2}\left(m^{\prime}, m\right)\right\}} P\left\{s_{t}=m \mid s_{t-1}=m^{\prime}\right\}
$$

Where $C_{i}^{j}$ is $d_{k}^{j}$ after interleaving.

In formula (7), $P\left(d_{k}^{j}\right)$ indicates the priority probability of $d_{k}^{j}$, if we consider 0 and 1 are transmitted in the same probability, we can consider $P\left(d_{k}^{j}=0\right)=P\left(d_{k}^{j}=1\right)=0.5$.

But to gain a better performance of turbo code, we can rewrite (7) in new form:

$$
P_{\text {dem }}\left(d_{k}^{1} \mid y_{k}\right)=\sum_{d_{k}^{1}, \ldots d_{k}^{2} \in\{, 1\}} \frac{P\left\{y \mid s\left(d_{k}^{1}, d_{k}^{2}, d_{k}^{3}, d_{k}^{4}\right)\right\}}{P\left(y_{k}\right)} \gamma_{\text {dec }}\left(d_{k}^{1}\right) \gamma_{\text {dec }}\left(d_{k}^{2}\right) \gamma_{\text {dec }}\left(d_{k}^{3}\right) \gamma_{\text {dec }}\left(d_{k}^{4}\right)
$$

Where $\gamma_{d e c}\left(d_{k}^{j}\right)$ replaces $P\left(d_{k}^{j}\right)$ and $\gamma_{d e c}\left(d_{k}^{j}\right)$ is the a priori probability of $d_{k}^{j}$. Now using the iteration between demodulator and decoder structure in figure 1 , we can get the extrinsic probability $\gamma_{d e c}\left(d_{k}^{j}\right)$ from the MAP decoder and send this extrinsic probability back to the demodulator as the a priori probability to improve the results. One more thing need be paid attention to is that (10) is optimal at gray mapping constellation, considering on hierarchical modulation, (10) should be re-formula as:

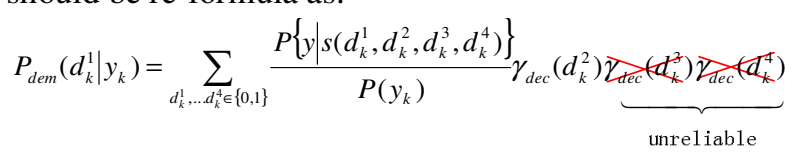

This formula is for Hp bits $d_{k}^{1}$ and $d_{k}^{2}$, and for Lp bits $d_{k}^{3}$ and $d_{k}^{4}$, it would be:

$$
P_{\text {dem }}\left(d_{k}^{3} \mid y_{k}\right)=\sum_{d_{k}^{1}, . d_{k}^{*} \in\{0,1\}} \frac{P\left\{y \mid s\left(d_{k}^{1}, d_{k}^{2}, d_{k}^{3}, d_{k}^{4}\right)\right\}}{P\left(y_{k}\right)} \underbrace{\gamma_{\text {dec }}\left(d_{k}^{1}\right) \gamma_{\text {dec }}\left(d_{k}^{2}\right)}_{\text {reliable }} \gamma_{\text {dec }}\left(d_{k}^{4}\right)
$$

$$
P_{d e m}\left(d_{k}^{1} \mid y_{k}\right) \text { and } P_{d e m}\left(d_{k}^{3} \mid y_{k}\right) \text { are derived } \mathrm{n}=\text { from (11) and }
$$
(12), the a priori probability of $d_{k}^{2}$ is same to that of $d_{k}^{1}, d_{k}^{4}$ are same to $d_{k}^{3}$.It can be depicted from (11), (12) that for the $\mathrm{Hp}$ bits, the extrinsic probability can be considered reliable so the probability can be used at the Lp decoding, but on the contrary, the extrinsic probability of Lp bits is not reliable so be discard at the decoding process of $\mathrm{Hp}$ bits.

\section{Simulation Results}

The system simulation is done at the ITPP simulation platform, using Monte-Carlo simulation method. The generator polynomial of the turbo encoder is $(23,35)_{8}$, constraint length is 5 , interleaving depth is 1024 , and the total package sent is 800 , so there are 1024 bits in every package. The turbo iteration time is 8 , and demodulation to decoding iteration time is 5, receiving symbols are passed through Rayleigh and AWGN channel. At first we consider on the BER performance of gray map constellation, Set Partitioning constellation and hierarchical modulation with $\alpha=1$ shown in figure 3 . from the figure we can depict that the hierarchical modulation has the best performance in the three constellation at low SNR, though after number of iterations and at higher SNR the other two can get better performance than they are at lower SNR. This result can indicate select hierarchical modulation may achieve a better performance at low SNR for the Lp bits.

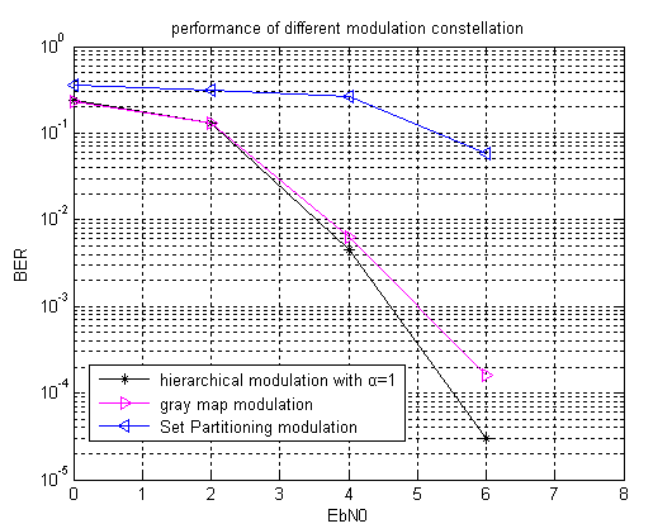

Figure.3 BER performance of different constellation mapping

Figure. 4 shows the BER performance of different $\alpha$ hierarchical modulation. The other parameters are the same to the above simulation. From the figure we can depict that when increase $\alpha$, the BER performance of Hp can get better but at the same time the BER of Lp is worse. We can choose a proper scheme according to the SNR in the center of a sector and the SNR at the margin of a sector.

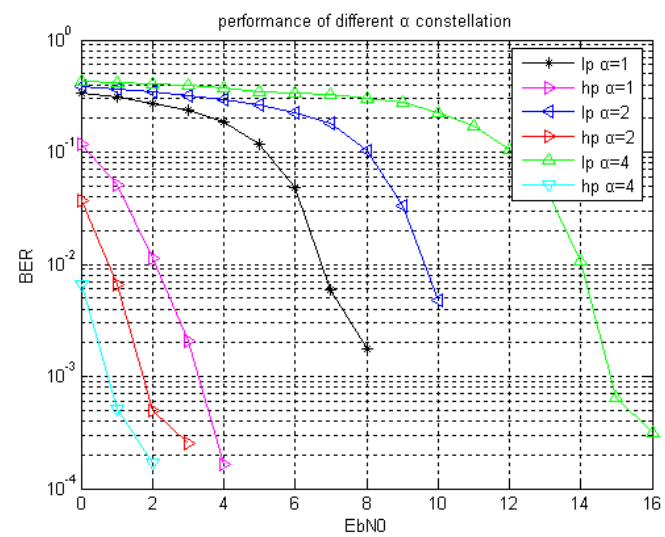

Fig. 4 BER of hierarchical modulation with different $\alpha$ 
Figure 5 shows the BER performance of new iteration scheme depicted in this paper. The constellation is hierarchical with $\alpha=1$. The other parameters are the same to the above. From the curve we can see that when increase the iteration times between the demodulator and the decoder can get a better performance about $0.5 \mathrm{~dB}$ when iteration time is 4 . But the performance do not increase highly when iteration time increase from 1 to 4 . So we can find an optimal iteration scheme is to do 1 iteration between demodulator and decoder first, then do the turbo decoding iteration. This scheme is shown in table 1 . If we use scheme 2 in the table to do the decode calculation we can save about 24 times iteration but get almost same results, if we want to get better BER we can use scheme 3 and 4, from the table we can depict these scheme do not increase the decoding algorithm more complexity, and can gain a better performance.

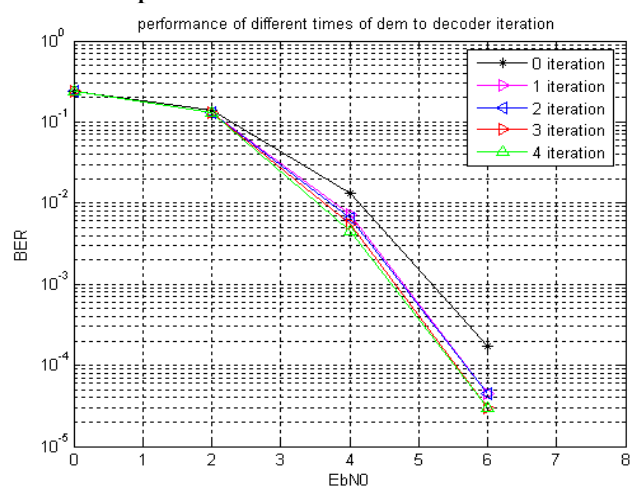

Figure.5 BER performance of different demodulation-decoder iterations

Table 1 iteration time scheme

\begin{tabular}{|c|c|c|c|c|}
\hline 0 & 40 & 8 & 8 & 8 \\
\hline 1 & 0 & 8 & 8 & 8 \\
\hline 2 & 0 & 0 & 8 & 8 \\
\hline 3 & 0 & 0 & 0 & 8 \\
\hline 4 & 0 & 0 & 0 & 0 \\
\hline $\begin{array}{c}\text { Total } \\
\text { iteration }\end{array}$ & 40 & 16 & 24 & 32 \\
\hline
\end{tabular}

(TI: turbo iteration ; DI: demodulation iteration)

\section{CONCLUSIONS}

In this paper, we discuss the new constellation mapping in the next generation communication scheme. And the new constellation can get better BER performance without adding complexity to the constellation as to the system. And according to the constellation style and system feature, there is a new iteration scheme brought forward. The new iteration scheme can make the system a $0.5 \mathrm{~dB}$ better BER performance without adding complexity. So this scheme can be used in the same level of the current hardware to be realized.

\section{REFERENCES}

[1] DVB bluebook, A111,Rev.1 July 2007

[2] 3GPP TR 25.814 V7.1.0.Physical layer aspects for evolved Universal Terrestrial Radio Access(UTRA)(Release 7)

[3] 3GPP R1-050765.Ericsson, Some Aspect of Single Carrier Transmission for E-UTRA, 3GPP TSG RAN WG1 Meeting\#42

[4] 3GPP R1-071211.RAN WG4, Motorola, Response to LS on FDSS(to: RAN1), 3GPP TSG RAN WG1 Meeting\#48

[5] Berrou C, Glavieux A , Thitimajshama, "Near Shannon limit errorcorrecting coding and decoding: turbo-codes" P. [C] Proceedings of IEEE International Conference on Communications.

[6] Branka Vucetic, JinHong Yuan, "turbo codes: principles and applications", 2000

[7] L.R.BAHL, J.COCKE, F.JELINEK, and J.RAVIV, "optimal decoding of linear codes for minimizing symbol error rate”, IEEE 1974.

[8] Jun Asatani, "Soft-Input Soft-Output Decoding Algorithm of Linear Block Codes Based on Minimum Distance Search", IEEE 2004

[9] William E. Ryan, "A turbo code tutorial”, Proc. IEEE Globecom, 1998

[10] Luc vandendorpe, "novel receiver and decoding schemes for wireless OFDM systems with cyclic prefix or zero padding”, 2001

[11] Schreckenbach. N Gortz. J Hagenauer.G Bauch., "Optimized Symbol Mappings for Bit-Interleaved Coded Modulation with Iterative Decoding”, Global Telecommunications Conference, 2003. GLOBECOM '03. IEEE, Vol. 6 (2003), pp. 3316-3320 vol.6 